mitochondrial disease.

\title{
GAUCHER DISEASE TYPE IIIC, WITH OCULOMOTOR APRAXIA
}

Four siblings with consanguineous parents, presenting with oculomotor apraxia in early childhood, were diagnosed with Gaucher disease (GD) at 10 years of age, at King Faisal Specialist Hospital, Riyadh, Saudi Arabia. Slow horizontal saccades, compensatory head thrust, and reading disability in early childhood was followed by cardiovascular calcification at 10 years. Bone marrow biopsy showed Gaucher cells, and cultured fibroblast B-glucocerebrosidase was reduced to $10 \%$ of control levels. Genotype analysis in 2 patients showed homozygosity for D409H $(1342 \mathrm{G}->\mathrm{C})$ mutation. The uniformity of symptoms and signs allowed classification of this variety of GD as the type IIIC. (Bohlega S, Kambouris M, Shahid M, Al Homsi M, Al Sous W. Gaucher disease with oculomotor apraxia and cardiovascular calcification (Gaucher type IIIC). Neurology Jan 2000;54:261-263). (Reprints: Dr Saeed Bohlega, Department of Neurosciences (MBC 76), King Faisal Specialist Hospital and Research Centre, PO Box 3354, Riyadh 11211, Saudi Arabia).

COMMENT. Oculomotor apraxia presenting in early childhood and progressive aortic and mitral valve calcification in adolescence are the distinctive clinical manifestations of Gaucher disease, type IIIC.

\section{NEUROMUSCULAR DISEASE}

\section{SURAL NERVE AND NERVE CONDUCTION STUDIES IN GUILLAIN- BARRE SYNDROME}

Sural nerve biopsy findings from 29 of 50 patients (median age, 4.75 yrs) with Guillain Barre syndrome (GBS), admitted to Beijing Children's Hospital, China, were correlated with nerve conduction studies, to determine the reliability of diagnosis of GBS subtypes. All 11 patients with acute motor axonal neuropathy (AMAN) had normal sural nerve biopsies, whereas all 3 cases classified electrophysiologically as acute inflammatory demyelinating polyneuropathy (AIDP) showed macrophage-mediated demyelination on biopsy. One patient with reduced sural sensory nerve action potentials and classified as acute motor sensory axonal neuropathy (AMSAN) had the greatest number of degenerating sensory nerve fibers on sural nerve biopsy. Nerve conduction studies are reliable in differentiating subtypes of GBS. (Lu JL, Sheikh KA, Wu HS et al. Physiologicpathologic correlation in Guillain-Barre syndrome in children. Neurology Jan 2000;54:33-39). (Reprints: Dr Tony W Ho, Department of Neurology, Johns Hopkins Hospital, Pathology 509, 600 N Wolfe St, Baltimore, MD 21287).

COMMENT. The reliability of classification of Guillain-Barre syndrome subtypes on the basis of nerve conduction studies (NCS) is confirmed by sural nerve biopsy findings. NCS alone may be sufficiently diagnostic.

Epidemiological, clinical, and electrodiagnostic features of GBS variants were studied in 61 children, aged 14 months to $14 \mathrm{yrs}$, admitted to the Hospital Nacional de Pediatria, Buenos Aires. Two groups were identified by electrodiagnosis: 18 with AMAN, and 43 with AIDP. Those with AMAN were younger and $90 \%$ lived outside the metropolitan area, in rural areas without running water. In contrast, $50 \%$ of children with AIDP resided in Buenos Aires City. Poor hygienic conditions underly the increased incidence of AMAN in underdeveloped countries. (Paradiso G, et al. Ann Neurol Nov 1999;46:701-707). 